\title{
Spermatozoa induce transcriptomic alterations in bovine oviductal epithelial cells prior to initial contact
}

\author{
Qurat UI Ain Reshi ${ }^{1}$ • Janeli Viil ${ }^{1}$ - James Ord ${ }^{1}$ • Freddy Lättekivi ${ }^{1}$ • Kasun Godakumara ${ }^{1}$. Mohammad Mehedi Hasan ${ }^{1}$. \\ Monika Nõmm ${ }^{2} \cdot$ Kersti Jääger $^{3} \cdot$ Agne Velthut-Meikas $^{4}$ Ülle Jaakma ${ }^{2} \cdot$ Andres Salumets $^{2,3,5,6} \cdot$ Alireza Fazeli $^{1,7}$ (B)
}

Received: 9 May 2020 / Accepted: 31 July 2020/Published online: 3 September 2020

(C) The Author(s) 2020

\begin{abstract}
The capability of spermatozoa to directly influence maternal gene expression is already established. Indeed, some of the changes induced by spermatozoa may have a direct functional importance in the pre-conceptional period. Although the mechanisms underlying these sperm-maternal interactions are not well characterized, it is possible that they could involve ligands that are released from the spermatozoa. This study therefore aimed to test whether physical contact between bovine spermatozoa and bovine oviductal epithelial cells (BOECs) is a prerequisite for spermatozoa-induced gene expression changes. We used two coculture models: a contact co-culture model in which spermatozoa interact directly with BOECs, and a non-contact co-culture model in which an insert with the pore size of $0.4 \mu \mathrm{m}$ was placed between spermatozoa and BOECs. Messenger RNA sequencing analysis of BOECs by RNA-seq revealed ten differentially expressed genes in contact system and 108 differentially expressed genes in the non-contact system after $10 \mathrm{~h}$ of co-culture. Retinol metabolism pathway and ovarian steroidogenesis pathway were significantly enriched in the non-contact co-culture system. Q-PCR analysis revealed that transcriptional responses can be rapid, with increased expression of four genes (DHRS3, CYP1B1, PTGS2, and ATF3) detectable within just 90 min of co-incubation, but with expression levels highly dependent on the type of co-culture system. The findings from our study demonstrate that direct contact with spermatozoa is not necessary to induce changes in gene expression of oviductal epithelial cells, suggesting that spermatozoa may be able to signal to maternal tissues in advance of their arrival.
\end{abstract}

Keywords Bovine oviductal epithelial cells $\cdot$ Spermatozoa $\cdot$ Contact co-culture $\cdot$ Non-contact co-culture

\section{Background}

The oviduct, also called fallopian tube in mammals, is an important site where several crucial cellular and molecular events have to occur between the cells of the female reproductive tract and gametes of both sexes to have a successful conception. The

Electronic supplementary material The online version of this article (https://doi.org/10.1007/s12079-020-00575-2) contains supplementary material, which is available to authorized users.

Alireza Fazeli

alireza.fazeli@ut.ee

1 Department of Pathophysiology, Institute of Biomedicine and Translational Medicine, University of Tartu, Ravila 14B, 50411 Tartu, Estonia

2 Institute of Veterinary Medicine and Animal Sciences, Estonian University of Life Sciences, Tartu, Estonia

3 Competence Centre on Health Technologies, Tartu, Estonia reproductive tract communicates and interacts with spermatozoa during the pre-conception period in diverse ways in order to facilitate their maturation, transportation, and survival, and establish proper physiological conditions for fertilization (Fazeli et al. 2004; Holt and Fazeli 2016; "The fallopian tubes," 1989). Spermatozoa interact with oviductal cells at two levels -
4 Department of Chemistry and Biotechnology, School of Science, Tallinn University of Technology, Tallinn, Estonia

5 Department of Obstetrics and Gynaecology, Institute of Clinical Medicine, University of Tartu, Tartu, Estonia

6 Institute of Genomics, University of Tartu, Tartu, Estonia

7 Academic Unit of Reproductive and Developmental Medicine, Department of Oncology and Metabolism, Medical School, University of Sheffield, Sheffield, UK 
physical contacts and molecular interactions. Physical contacts include sperm cells swimming through the female reproductive tract aided by the flow of the oviductal fluid, the ciliary beating of the epithelial cells, and contractions of the female reproductive tract. On the molecular level, spermatozoa interact with oviductal cells through their surface proteins that have been shown to alter the gene expression of the epithelial cells in the oviduct (Alvarez-Rodriguez et al. 2019; Fazeli et al. 2004; López-Úbeda et al. 2015). Oviduct cells produce a diverse range of secreted proteins, extracellular vesicles $(\mathrm{EV})$ and other specific signaling molecules, some of which are implicated in sperm-oviduct interactions (Almiñana and Bauersachs 2019; Jamaludin et al. 2019). However, less is known about the signaling molecules released from spermatozoa that affect epithelial cells of the female reproductive tract.

Several in vivo studies have reported that oviduct responds to the arrival of spermatozoa through changes in its transcriptome as well as in other morphological processes including ciliary beating and secretion of bioactive compounds which mediate transport of the sperm cells (Almiñana and Bauersachs 2019; Fazeli et al. 2004; Kodithuwakku et al. 2007). In a study conducted on female turkeys, it was found that $1 \%$ of the genes in the sperm storage tubules were differentially expressed $48 \mathrm{~h}$ after sperm insemination suggesting that sperm caused alterations in the gene expression of the female reproductive tract (Long et al. 2003). Similar studies in pigs have shown that several genes in sow's oviductal cells are differentially expressed between naturally inseminated and non-inseminated animals (López-Úbeda et al. 2015). Another piece of evidence supporting the idea of spermatozoa affecting the gene expression of oviductal cells came from a study where authors used mutated mice (T145H mutation) with spermatogenic arrest, which are only able to produce seminal plasma but not spermatozoa. When such infertile male mice were mated with normal female mice, no changes in expression levels were detected in adrenomedullin and prostaglandin-endoperoxidase synthase 2 (PTGS2) genes but after mating with normal male mice both of these genes were up-regulated suggesting that sperm cells indeed alter the expression of specific genes in oviductal epithelium (Fazeli et al. 2004).

Studies suggest that the entry of spermatozoa to the reproductive tract elicits a uterine inflammatory response and therefore alters the innate as well as the acquired local immune system (Rodríguez-Martínez et al. 2009; Rozeboom et al. 1998). Natural or artificial insemination in the cervix or upper reproductive tract in porcine females has been shown to modify, mostly down-regulate, the expression of various genes responsible for modulating the local immune response, including chemokine and interferon-gamma signaling genes, and JAK/STAT pathway-related genes (Alvarez-Rodriguez et al. 2019). Furthermore, after mating the gene expression of cytokine related genes such as TNFSF11 (TNF superfamily member 11) and ADGRB2 (Adhesion G Protein-
Coupled Receptor B2) was also down-regulated (AlvarezRodriguez et al. 2019), a response usually elicited to eliminate invading microorganisms. Therefore, the mechanisms of sperm tolerance and survival appear to be counteracting such inflammatory responses, although their extensive details are yet to be discovered.

Although it has been established that spermatozoa induce changes in the gene expression of the oviductal epithelium, it has remained mostly unknown by which mechanisms spermatozoa communicate/interact with epithelial cells to elicit such responses. It is also not fully known what type of molecular receptors or contact points between spermatozoa and reproductive tract epithelium are necessary. Among various communicating agents and messenger molecules, EVs that contain a mixture of proteins, peptides, miRNAs, lipids as well as DNA fragments are suggested to play an important role in this inter-cell communication (Almiñana and Bauersachs 2019; Ferraz et al. 2019). Several studies have shown that seminal plasma contains diverse types of EVs such as prostasomes, small vesicles secreted by prostatic epithelial cells, which are important for sperm hyper-motility and acrosome reaction (Aalberts et al. 2013). Prostasomes bind to spermatozoa in the uterus and are carried into the oviduct in association with spermatozoa (Aalberts et al. 2013), however, it is equally likely that prostasomes may be involved in sperm-oviduct dialogue, as well. It is still unknown if spermatozoa themselves release EVs, which could mediate communication with oviductal cells by modulating their gene expression.

The current study was set out to investigate whether mature spermatozoa release biomolecules that could trigger gene expression changes in oviductal cells by using contact and noncontact co-culture systems with bovine spermatozoa and primary bovine oviductal epithelial cells (BOECs). For this study we used a novel non-contact co-culture system to analyse whether the spermatozoa could communicate with oviductal epithelial cells, when not in direct contact with them.

\section{Materials and methods}

\section{Isolation and culture of primary BOECs}

Oviducts with attached ovaries were collected from the slaughterhouse and transported to the laboratory in saline at $37^{\circ} \mathrm{C}$ within $4 \mathrm{~h}$ after animal slaughter. Only the oviducts of the early stage of the estrous cycle (bright red corpus luteum, 0-4 days after ovulation) were selected for isolation (Ireland et al. 1980). The ovary and the connective tissue were removed from the oviducts with a scalpel, followed by washing with wash buffer-1 (DPBS supplemented with Amphotericin B $(1 \mu \mathrm{l} / \mathrm{ml})$ and $1 X$ Penicillin/Streptomycin $(10 \mu \mathrm{l} / \mathrm{ml}))$. The ampulla and the isthmus part of the oviducts were separated by cutting the oviducts at the ampullary-isthmic junction and 
were handled separately. The mucosa was extracted by squeezing the oviduct gently with a sterile glass slide and transferred into a tube containing wash buffer-1. The cells were washed thrice, two times in wash buffer- 1 and once in wash buffer-2 (DPBS supplemented with Amphotericin B (1 $\mu \mathrm{l} / \mathrm{ml})$, Penicillin/Streptomycin $(10 \mu \mathrm{l} / \mathrm{ml})$ and $5 \% \mathrm{FBS})$ and between each wash, the cells were allowed to settle down after which the supernatant was removed. The final pellet of the cells was resuspended in culture media (DMEM/F12 supplemented with $10 \% \mathrm{FBS}$, Amphotericin B $(1 \mu \mathrm{l} / \mathrm{ml})$ and Penicillin/Streptomycin $(10 \mu \mathrm{l} / \mathrm{ml}))$, transferred to T-25 flasks and incubated in a humidified atmosphere with $5 \% \mathrm{CO}_{2}$ at $38.8^{\circ} \mathrm{C}$. The cells were allowed to attach for the next 3 days without changing the media. Afterwards, the cells were trypsinized and transferred to a T-75 flask, and media was changed every $48 \mathrm{~h}$ until confluency of $80 \%$ was attained. The cells had been split four times before they were cocultured with spermatozoa.

\section{Immunofluorescence analysis of bovine epithelial cells (BOECs)}

BOECs were grown on coverslips and then fixed with $4 \%$ paraformaldehyde for $15 \mathrm{~min}$ at room temperature, and in order to permeabilize the cell membrane, cells were treated with cold methanol for $10 \mathrm{~min}$ on ice. Thereafter, $4 \%$ normal goat serum was used for blocking for $1 \mathrm{~h}$ at room temperature. The cells were then incubated with a mix of anti-Cytokeratin (C2562, 1:250, Sigma-Aldrich) and antiVimentin (PLA0199, 1:250, Sigma-Aldrich, USA) primary antibodies in blocking buffer overnight at $4{ }^{\circ} \mathrm{C}$. Negative control was incubated with a blocking buffer lacking any of the primary antibodies. Secondary antibody incubation was done using goat anti-mouse (conjugated to Alexa Fluor 488,1:500) and goat anti-rabbit (conjugated to Alexa Fluor 594, 1:500) secondary antibodies (both Invitrogen, Thermo Fisher Scientific, Eugene, USA) in blocking buffer for $1 \mathrm{~h}$ in the dark at room temperature. After incubation, the nuclei were stained using Hoechst 33342 (1:2000, Thermo Fisher Scientific) for $3 \mathrm{~min}$ and then the coverslips were mounted with Fluorescence Mounting Medium (Dako, Denmark). Images were taken with a Leica DM5500B microscope equipped with Leica DFC310 camera (Leica, Wetzlar, Germany) and processed with ImageJ (Schindelin et al. 2012).

\section{Spermatozoa washing and culture}

Within each of the two experiments (RNAseq and qPCR), we used multiple semen straws all deriving from the same ejaculate from the same bull. For each day that the experiment was carried out, nine straws of frozen bovine semen were thawed in $37{ }^{\circ} \mathrm{C}$ sterile water bath for $30 \mathrm{~s}$. Contents of the three straws $(250 \mu \mathrm{l}$ of semen in each straw) were pooled and deposited onto $4 \mathrm{ml}$ of $60 \%$ isoosmotic Percoll® solution (GE Healthcare, 17-0891-02, Sweden) prepared as described previously (Aldarmahi et al. 2014), with slight modifications. The modifications included making $100 \%$ Percoll by mixing $1 \mathrm{X}$ HEPES with Percoll in 1:9 ratio, while $60 \%$ Percoll was prepared by diluting $100 \%$ Percoll with supplemented SpermTALP media. Percoll with semen was centrifuged for $20 \mathrm{~min}$ at $300 \times \mathrm{g}$ at room temperature and the pellet was washed with pre-warmed EV-depleted Sperm-TALP (NaCl: $0.005 \mathrm{~g}$ / $\mathrm{ml}, \mathrm{KCl}: 0.23 \mathrm{mg} / \mathrm{ml}, \mathrm{NaH}_{2} \mathrm{CO}_{3}: 0.002 \mathrm{~g} / \mathrm{ml}$, lactic acid $60 \%$ $/ \mathrm{ml}, \mathrm{NaH}_{2} \mathrm{PO}_{4}: 0.034 \mathrm{mg} / \mathrm{ml}, \mathrm{CaCl}_{2}: 0.308 \mathrm{mg} / \mathrm{ml}, \mathrm{MgCl}_{2}$ : $100 \mathrm{mM} 15 \mathrm{ul} / \mathrm{ml}$, HEPES: $0.0023 \mathrm{~g} / \mathrm{ml}$, Gentamycin 0.5ul/ $\mathrm{ml}$, sodium pyruvate: $0.1 \mathrm{ul} / \mathrm{ml}$, and BSA: $0.6 \mathrm{mg} / \mathrm{ml}$ ) media and centrifuged again for $5 \mathrm{~min}$ at $400 \times \mathrm{g}$. Due to the presence of BSA (AppliChem, A1391, 0050, Germany) in the media, $\mathrm{EV}$ depletion was performed in order to minimize the number of BSA-derived particles in the media by filtering the BSA solution through $100 \mathrm{kDa}$ Amicon® Ultra-15 Centrifugal Filter Unit (R9CA01172, Ireland) (Kornilov et al. 2018). The final pellet was resuspended in $1 \mathrm{ml}$ of pre-warmed EVdepleted Sperm-TALP media and the concentration of washed spermatozoa was determined. The concentration was adjusted to $1 \times 10^{6} / \mathrm{ml}$ and the motility was analysed in five different fields under the microscope with $40 \mathrm{X}$ magnification. Spermatozoa were processed on three different days for RNA sequencing experiment and the measured average motility post-thawing was 60\% (day-1), 65\% (day-2) and 60\% (day-3). Similarly for experiments with q-PCR, the average motility of spermatozoa on three different days was $65 \%$ (day1), $70 \%$ (day-2) and $60 \%$ (day-3) post-thawing. All procedures were performed under aseptic conditions and the washed spermatozoa were immediately used for co-culturing with BOECs.

\section{Co-culturing BOECs and spermatozoa in a contact and non-contact co-culture model}

Frozen BOECs from isthmus and ampulla were thawed and first cultured separately. Afterwards, the cells from ampulla and isthmus were mixed and plated onto a 12-well plate until they attained $80 \%$ confluency, at which point cells were divided across experimental replicates. Before the addition of spermatozoa, the BOECs were washed once with pure DMEM/F12 media, followed by washing with EV depleted sperm-TALP media.

In order to identify the genes that were differently expressed in BOECs in response to spermatozoa, we used two co-culture models and an independent BOEC culture as the control group. In the first co-culture model, the spermatozoa were allowed to directly interact with BOECs - hereinafter referred to as contact co-culture. In the second co-culture model, an insert with a pore size of $0.4 \mu \mathrm{m}$ (Thincert cell culture 
insert, Greiner Bio-One GmbH, Kremsmünster, Austria) was used to spatially separate the spermatozoa and BOECs - hereinafter referred to as non-contact co-culture. Equal amounts of washed bovine spermatozoa $\left(1 \times 10^{6}\right.$ spermatozoa $\left./ \mathrm{ml}\right)$ were added to BOECs in both co-culture models and coincubation was performed for $10 \mathrm{~h}$, after which RNA was extracted from the BOECs for sequencing. The control group BOECs were processed equivalently. The aforementioned experiment was repeated three times on three different days, using a different aliquot of the same primary cells. The source of spermatozoa was also kept constant (all thawed semen straws derived from the same ejaculate). In each repetition of the experiment, both co-culture models and the control group were cultured across three replicate wells. After the three repetitions of the experiment, the RNA from the wells was pooled across all three repetitions of the experiment, so that a final experimental replicate consisted of three pooled wells, each of which originated from a different repetition of the experiment. The pooling of wells across the experimental repetitions was conducted in order to alleviate batch variation as it was not possible to perform the whole experiment with all replicates in a single day. The experimental design is explained in more detail in Fig. 1.

In order to unfold the dynamics of gene expression change for the selected genes of interest, additional experiments were performed using the same co-culturing conditions with varying incubation periods during which the spermatozoa were allowed to exert an effect on the BOECs. The same source of BOECs as in the RNA sequencing experiment was used. The spermatozoa, however, originated from a different ejaculate of the same bull. The experiment was repeated for four different coincubation periods for both the co-culture models and the control BOECs. The time periods were $30 \mathrm{~min}, 90 \mathrm{~min}$, $2.5 \mathrm{~h}$, and $4 \mathrm{~h}$. The experiments were conducted in three

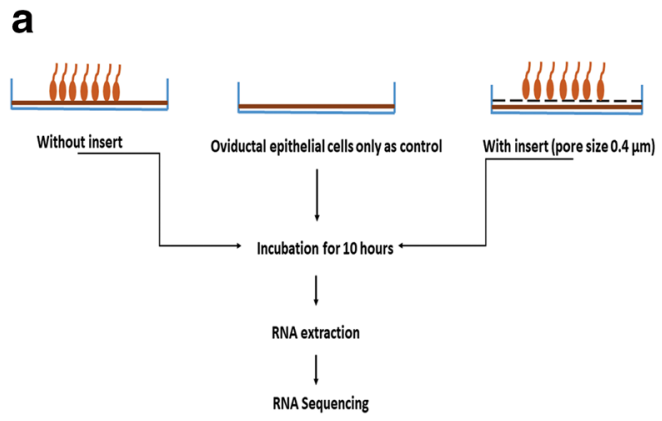

Fig. 1 a Schematic illustration of the two co-culture models used in the experimental set up where in one model the spermatozoa interact directly with the BOECs monolayer (contact co-culture) and in other model an insert with a pore size of $0.4 \mu \mathrm{m}$ was used to separate BOECs from spermatozoa (non-contact co-culture). BOEC monolayer with no spermatozoa identical repetitions on three different days resulting in three replicates for each experimental group.

\section{RNA extraction, sequencing library preparation, and RNA sequencing}

After the respective co-incubation periods, the media was discarded, followed by RNA extraction using QIAzol® Reagent (Qiagen, 79,306, USA) and isopropanol precipitation method, according to the manufacturer's protocol. Briefly, $500 \mu \mathrm{l}$ of guanidinium thiocyanate (QIAzol@ reagent) was added to BOECs in 12-well plate and the cells were left at room temperature for $10 \mathrm{~min}$. The contents were thoroughly homologized with a pipette, transferred to a clean tube and $100 \mu \mathrm{l}$ of chloroform was added. Once the samples were vortexed for $15 \mathrm{~s}$, they were immediately centrifuged at $12,000 \times \mathrm{g}$ for $15 \mathrm{~min}$ at $4{ }^{\circ} \mathrm{C}$ for phase separation. The aqueous phase containing RNA was transferred to a new tube and RNA was precipitated by adding $250 \mu \mathrm{l}$ of isopropanol to the aqueous phase and incubating samples at room temperature for $20 \mathrm{~min}$. Samples were centrifuged for $30 \mathrm{~min}$ at $16,000 \times \mathrm{g}$ at $4{ }^{\circ} \mathrm{C}$ and then the RNA pellet was washed thrice in $500 \mu \mathrm{l}$ of $75 \%$ ethanol. The final RNA pellet was air dried, resuspended in $30 \mu \mathrm{l}$ of nuclease free water and heated at $60{ }^{\circ} \mathrm{C}$ for $10 \mathrm{~min}$.

The extracted RNA was quantified using Qubit ${ }^{\mathrm{TM}} \mathrm{RNA} H \mathrm{H}$ Assay Kit according to the manufacturer's protocol (Q32852, ThermoFisher Scientific). The quality of the extracted RNA was determined with the Bioanalyzer Automated Electrophoresis instrument (Agilent technologies, Santa Clara, CA) and Agilent RNA 6000 nano Kit (Agilent technologies).

Smart-seq 2 methodology (Picelli et al. 2014) with slight modifications was employed to generate RNA sequencing libraries. Instead of single cells, we used $20 \mathrm{ng}$ of total RNA for cDNA synthesis and 10 cycles of PCR for pre-amplification. KAPA HiFi DNA polymerasewas replaced with Phusion

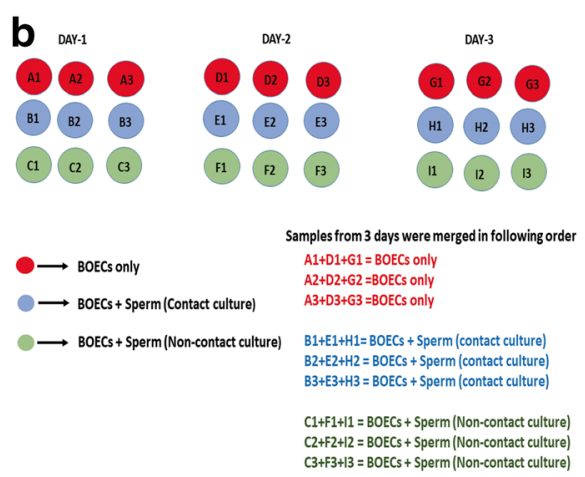

served as the control for the experiment. b Schematic diagram representing the number of replicates for each group and the procedure for merging the samples for RNA sequencing, RNA obtained was mixed across the three days to pool the variation arising from different days 
High-Fidelity DNA Polymerase (Thermo Scientific) which is compatible with the original protocol. $2 \mu$ l of diluted cDNA was applied to dual-index library preparation using Illumina Nextera XT DNA Sample Preparation Kit (FC-131-1024). Ampure XP beads (Beckman Coulter) were used for all clean-up steps and for size selection of 200-700 bp. All samples were pooled into a single library by equal concentration and sequenced on Illumina NextSeq500 using High Output Flow Cell v2.5 (single-end, 75 bp).

\section{Read processing, alignment, and counting for RNA- seq data analysis}

Read quality was assessed with FASTQC (Brown et al. 2017), trimming of reads for adapter sequences and the removal of low-quality reads was done using trimmomatic (Bolger et al. 2014). Reads were aligned to the $B$. taurus genome assembly (ARS-UCD1.2). Hisat2 was used for read alignment to the reference genome (Kim et al. 2019). Read alignment was performed with default parameters and with the inclusion of splice site information derived from the corresponding Ensembl B. taurus annotation file (ARS-UCD version 1.2.97). Gene-level read counts were obtained using featureCounts (Liao et al. 2014) with default parameters, using the Ensembl B. taurus annotation file (version 1.2.97) for the ARS-UCD1.2 genome assembly to obtain feature information.

\section{Differential expression analysis}

Differential expression analysis of gene-level counts was carried out in $\mathrm{R}$ version 3.6.1 using the edge $\mathrm{R}$ package (Robinson et al. 2010). The genes were filtered to exclude genes that were assigned less than 10 reads for all the samples in one experimental group. The genes remaining in the analysis were subjected to differential expression testing. Tagwise dispersion estimates were obtained based on the trended dispersions, and statistical comparisons were performed using a generalised linear model followed by likelihood ratio tests. The $P$ value was adjusted for multiple testing to obtain the false discovery rate (FDR) value using the default Benjamini-Hochberg method (Benjamini and Hochberg 1995). The results were considered statistically significant at false discovery rate $(\mathrm{FDR}) \leq 0.05$.

\section{Pathway enrichment analysis}

Pathway over-representation analysis was carried out using Rpackage clusterProfiler (Yu et al. 2012) and KEGG Pathway database annotations. Pathway analyses were carried out separately for up- and down-regulated genes which were differentially expressed at FDR $\leq 0.05$.

\section{Quantitative reverse transcription-PCR}

cDNA was synthesized using a mixture of oligo(dT) and random primers (FIREScript RT cDNA synthesis kit, Solis BioDyne, Tartu, Estonia). q-PCR was performed in triplicates for each biological sample and the cDNA products were amplified using the EvaGreen assay system (Solis BioDyne, Tartu, Estonia). The following program was used for amplification: $95^{\circ} \mathrm{C}$ for $15 \mathrm{~min}$, followed by 40 cycles of $95^{\circ} \mathrm{C}$ for $20 \mathrm{~s}, 62{ }^{\circ} \mathrm{C}$ for $20 \mathrm{~s}$ and $72{ }^{\circ} \mathrm{C}$ for $20 \mathrm{~s}$. A dissociation curve was performed to verify the purity of the PCR product. q-PCR data was analysed using a comparative CT method and the relative expression of RNA was calculated based on the $2^{-\Delta \Delta C T}$ method (Winer et al. 1999). Beta-2-microglobulin $(B 2 M)$ and TATA-binding protein $(T B P)$ genes were used for normalisation. Primers were designed using PrimerBLAST and the preference was given to the sequences that spanned exon-exon junction. Primer sequences for all genes can be found in Table 1.

\section{Statistical analysis and visualization}

Statistical analyses of qPCR-derived gene expression results were carried out in $\mathrm{R}$ version 3.6.1. For each gene of interest, a linear mixed model was fitted using the lmer function from the lme4 package (Bates et al. 2015). For each model, treatment and time were included as interacting fixed effect terms and experimental batch was included as a random effect to account for the collection of samples from three experimental batches. Inter-group comparisons at specific timepoints were performed as post-hoc pairwise comparisons of estimated marginal means with Tukey correction for multiple testing, for which the emmeans function from the emmeans package was used. All graphs were generated using ggplot 2 version 3.2.0 (Wickham 2009), except for the Venn diagram, for which the GOplot package version 1.02 was used (Walter et al. 2015).

\section{Results}

\section{Immunocytochemical localization of epithelial markers in BOECs}

Cytokeratins are important and major intermediate filaments of epithelial cells while Vimentin is the intermediate filament expressed in fibroblasts (Zeisberg and Neilson 2009). We used antibodies against cytokeratin and vimentin to evaluate if the primary cell culture we had established consisted mostly of epithelial cells, without fibroblast contamination. Incubation of the isolated cells with anti-Cytokeratin antibody showed a strong positive signal (Fig. 2a), whereas staining 
Table 1 Primer list for q-PCR

\begin{tabular}{ll}
\hline Gene name & Primer sequence $\left(5^{\prime}-3^{\prime}\right)$ \\
\hline PTGS2 & Forward: TGAGGAACTTACAGGAGAGAAGGA \\
CYP1B1 & Reverse: TCTACCAGAAGGGCGGGATA \\
& Forward: GGCTGACTCTGGCGATGGT \\
DHRS3 & Reverse: CTGCACTTCCGAATACCTGGTG \\
& Forward: TATTTCCGGGATGGTCTGTGC \\
ATF3 & Reverse: TATATTCCTGCCGTTCAACCAGT \\
& Forward: AGTGGATACAGGAGCAAAATGATG \\
Beta-2-microglobulin & Reverse: CAGAGGCACTGACTTCCGAG \\
& Forward: CTGCAAGGATGGCTCGCTT \\
TATA binding protein & Reverse: GAATCTTTGGAGGACGCTGGA \\
& Forward: GCACAGGAGCCAAGAGTGAA \\
& Reverse: TCCCCACCATGTTCTGAATCTT
\end{tabular}

with anti-Vimentin antibody produced a fade signal which confirmed the presence of epithelial cells mostly.

\section{RNA-seq results and differential gene expression}

Sequencing of the mRNA libraries yielded $6.2 \pm 1.2$ million reads (mean $\pm \mathrm{SD}$ ) per sample. After filtering for read quality, $98.7 \pm 0.2 \%$ of the reads remained, out of which $96.3 \pm 0.2 \%$ aligned to the $B$. taurus genome assembly (ARS-UCD1.2) at least once. Read counts were summarized at the gene level, and after filtering to remove genes considered not to be expressed in any of the groups, 10,636 genes remained and were subsequently tested for differential expression.

Non-contact co-culture model samples completely segregated from both control and contact co-culture model samples on the first principal component axis but exhibited considerably more noticeable intra-group variation compared to the other two groups (Fig. 3a). Contact co-culture with spermatozoa induced only minor changes to gene expression profiles of BOECs, with nine genes upregulated and one gene down-regulated (Fig. 3b). Complete list of differentially expressed (DE) genes in contact co-culture can be found in Supplementary Data (Table SI). The most upregulated gene in response to contact co-culture was $D H R S 3$, while the only downregulated gene was RANBP3 (Fig. 4a). Surprisingly, the non-contact co-culture treatment induced more extensive gene expression changes in BOECs (52 genes upregulated, and 56 genes downregulated; Fig. 3b). List of DE genes in non-contact co-culture can be found in supplementary data (Table SII). The topmost upregulated genes in response to non-contact co-culture were metabolic enzyme genes, including the mono-oxidases CYP1A1 and CYP1B1, as well as TXNRD1, DHRS3, and PTGS2 (Fig. 4b).

Four upregulated genes (PLAU, TNFRSF11B, SCIN, and $D H R S 3)$ were common to both contact and non-contact coculture (Fig. 4c). Of the four common upregulated genes,

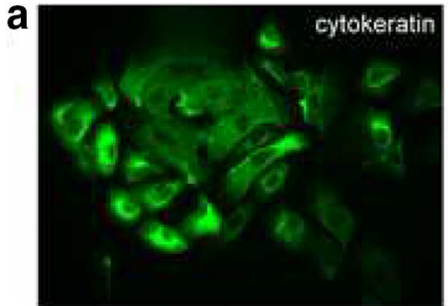

b
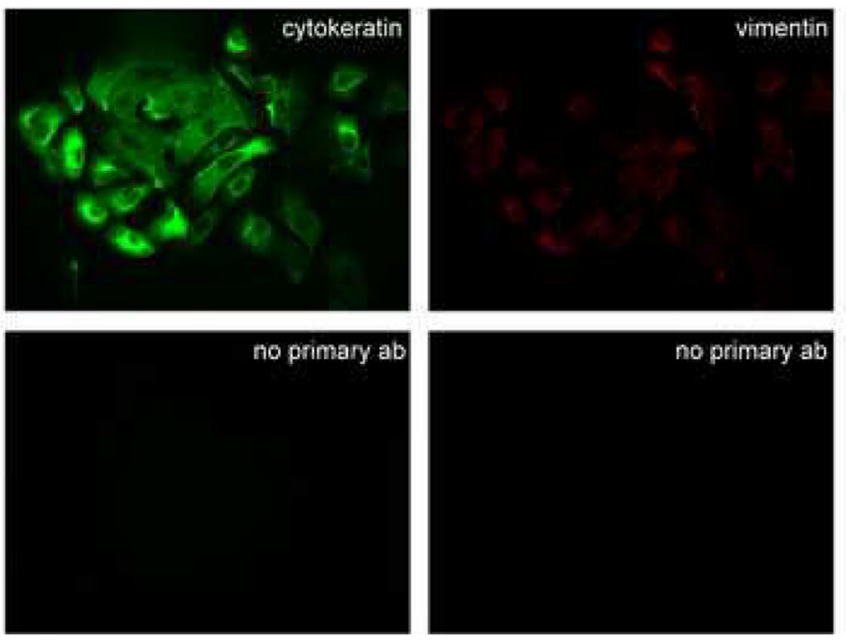

Fig. 2 Cytokeratin and Vimentin expression in primary bovine oviductal epithelial cells. a Cells displaying strong signal after staining with antiCytokeratin antibody (green) and faded signal with anti-Vimentin
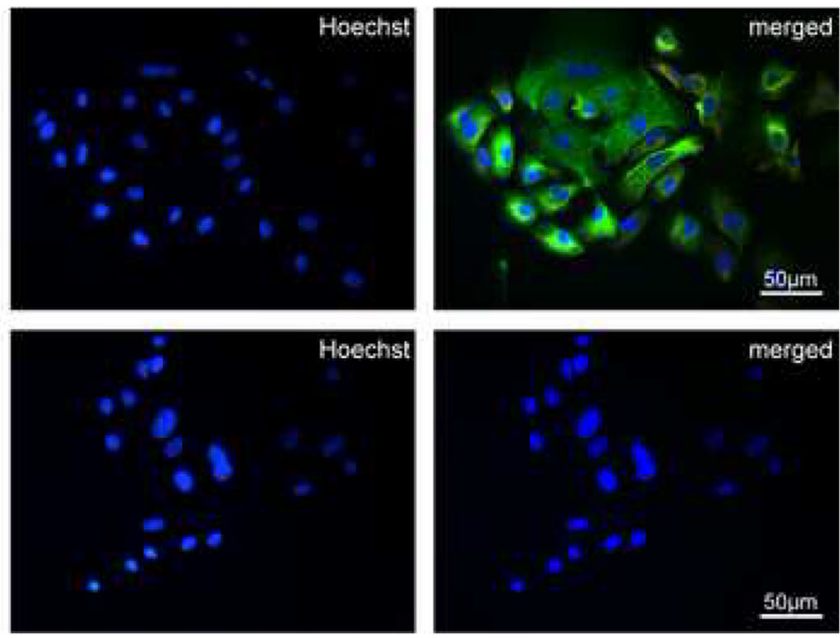

antibody (red). Nuclei were stained with Hoechst (blue). b Negative control staining without primary antibodies. Nuclei were stained with Hoechst 
a

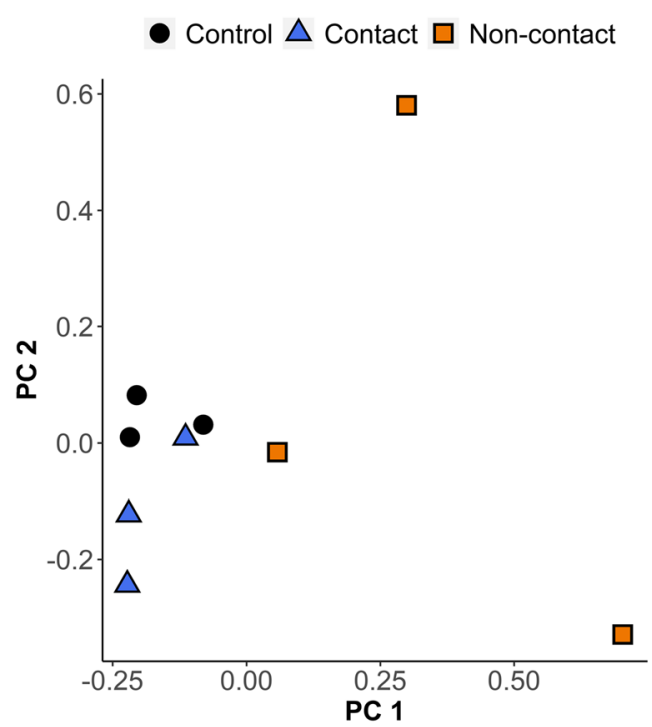

Fig. 3 a Principal Component Analysis (PCA) plot of inter-sample distances calculated based on $\log 2$ fold changes in the top 500 most variably expressed genes in BOECs under control conditions (black), contact coculture with spermatozoa (blue) and non-contact co-culture with spermatozoa (orange) after $10 \mathrm{~h}$ of incubation. b Heatmap of standardised (z- b

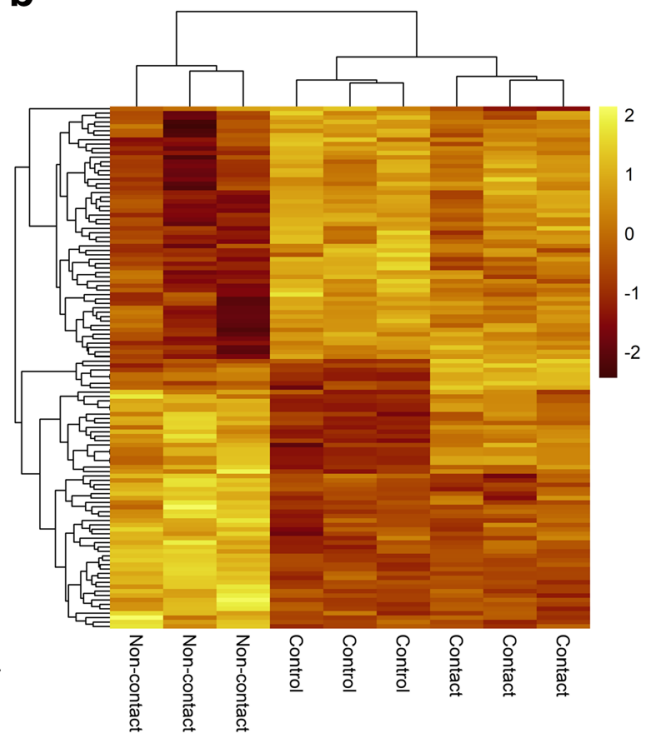

score) CPM values of genes that were differentially expressed either in response to contact or non-contact co-culture with spermatozoa, with lighter shade denoting higher levels of relative expression and darker shades denoting lower levels of relative expression
Fig. 4 a Volcano plot of the differentially expressed genes in BOECs in the contact co-culture model; the genes that were found to be statistically significant (FDR $\leq$ 0.05 ) are highlighted. b Volcano plot of the differentially expressed genes in BOECs in the non-contact co-culture model; the genes that were found to be statistically significant $(\mathrm{FDR} \leq 0.05)$ are

highlighted. $\mathbf{c}$ The number of statistically significant $(\mathrm{FDR} \leq 0.05)$ differentially expressed genes detected exclusively in either of the co-culture models or common to both of the model systems. The number of genes is presented on blue or orange backgrounds for downregulated or upregulated genes, respectively. The number of contra-regulated genes is presented on the greed background. $\mathbf{d}$ Network plot based on the pathways detected to be significantly enriched among the upregulated genes in BOECs in the non-contact co-culture model and the genes which contributed to the enrichment of these pathways

\section{a}

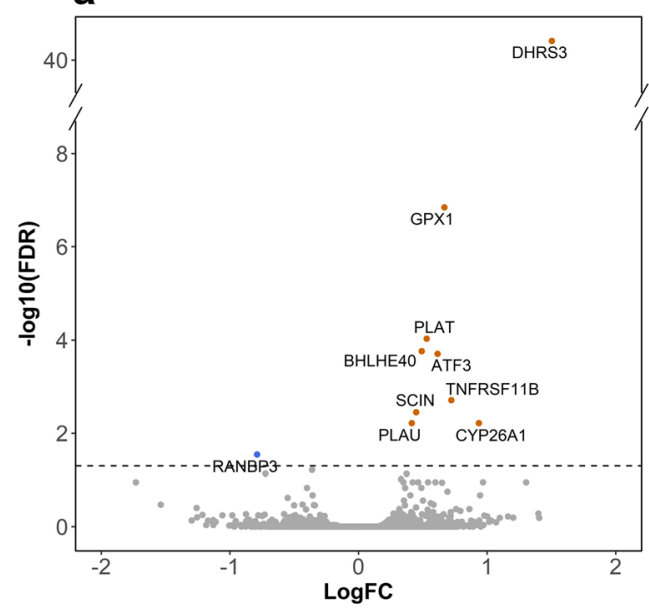

C

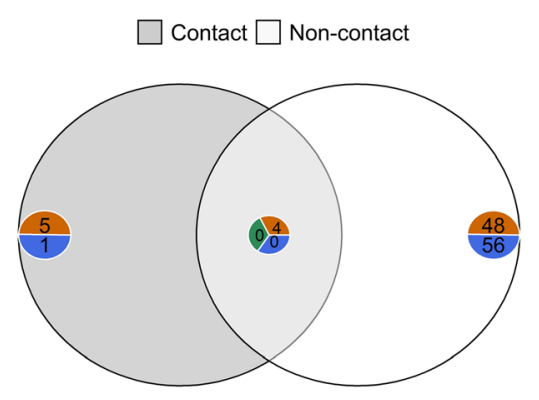

b

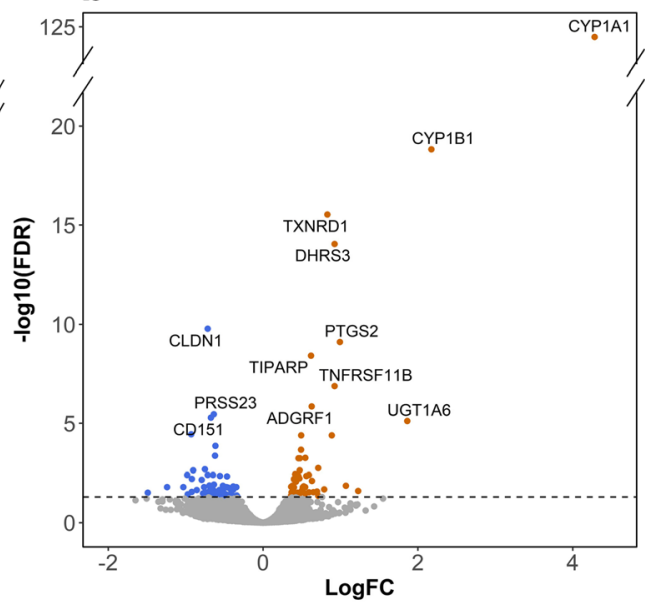

d

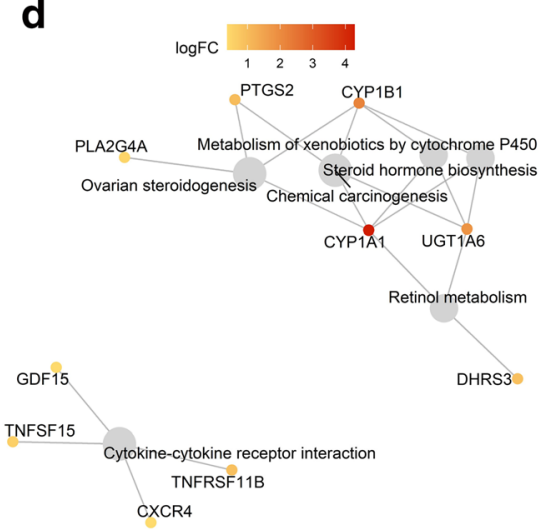


DHRS3 exhibited a higher degree of upregulation in contact co-culture $\left(\log _{2} \mathrm{FC}=1.5\right)$ compared to non-contact co-culture $\left(\log _{2} \mathrm{FC}=0.92\right)$. The other six DE genes in response to contact co-culture were unique to this treatment and interestingly included $A T F 3$, a known negative regulator of PTGS2 (Hellmann et al. 2015).

\section{Pathway over-representation analysis (non-contact co-culture)}

To identify pathways that were over-represented among genes that were differentially expressed in BOECs in response to non-contact co-culture with spermatozoa, pathway overrepresentation analyses was conducted separately for significantly up-regulated and down-regulated genes based on KEGG pathway annotations for B. taurus.

Among up-regulated genes, six pathways were significantly enriched at FDR $<0.05$, of which the most pertinent were retinol metabolism (bta00830), steroid hormone biosynthesis (bta00140), ovarian steroidogenesis (bta04913), and cytokine-cytokine receptor interaction (bta04060) (Table 2). The topmost enriched pathways also featured some of the most strongly upregulated genes: five featured CYP1A1, four featured $C Y P 1 B 1$, and two featured PTGS2 (Fig. 4d). No significantly enriched pathways were detected among downregulated genes.

\section{Short-term expression patterns of genes affected by contact and non-contact co-culture (qPCR)}

To examine how rapidly the BOECs respond to the stimuli of spermatozoa by changing the gene expression, we performed q-PCR analysis of four genes of interest for both contact and non-contact co-culture models after 30, 90, 150, and $240 \mathrm{~min}$ of co-culture. Specifically, we examined the expression of one gene shown to be up-regulated by RNA-seq in both treatments (DHRS3), two up-regulated genes only in response to noncontact co-culture (CYPIB1 and PTGS2), and one upregulated gene only in response to contact co-culture (ATF3).
DHRS3 showed increased expression in response to both contact and non-contact co-culture (Fig. 5a), but was stronger in response to contact, in which a statistically significant increase in expression was detectable starting from $30 \mathrm{~min}(P=$ 0.02 ) and a highly prominent increase observed at $240 \mathrm{~min}$. In contrast, a significant increase in DHRS3 in response to noncontact culture was not observed until $240 \mathrm{~min}$ had elapsed. DHRS3 expression was analysed following the removal of a prominent outlier in the non-contact group (2nd replicate, $90 \mathrm{~min})$.

Interestingly, the expression of $C Y P 1 B 1$ was prominently increased in response to non-contact after $90 \min (P<0.001)$, but the expression returned to the level of control samples at 240 min (Fig. 5b). Similarly DHRS3, CYP1B1 expression showed a weaker increase in response to contact co-culture which peaked at $150 \mathrm{~min}(P=0.04)$ and subsided afterwards.

$P T G S 2$ expression was similarly increased at $30 \mathrm{~min}$ in response to non-contact $(P=0.003)$, but conversely showing a sustained increase in expression levels of PTGS2 until the final timepoint. In contact co-culture, PTGS2 showed a modest increase in response to spermatozoa observable at $90 \mathrm{~min}$ $(P=0.02)$, but this increase in the expression levels of the gene was not sustained until the final timepoint (Fig. 5c).

ATF3 expression (Fig. 5d) was increased at $30 \mathrm{~min}$ in response to both contact $(\mathrm{P}<0.001)$ and non-contact co-culture $(\mathrm{P}<0.001)$. However, the expression of ATF3 was not increased at any other subsequent timepoint in non-contact coculture but remained significantly increased in response to contact co-culture until and including $150 \mathrm{~min}$ timepoint $(\mathrm{P}=0.04)$ before subsiding.

\section{Discussion}

While the capacity of spermatozoa to directly exert changes in maternal physiology has been known for some time (Fazeli 2011; Fazeli et al. 2004; Kodithuwakku et al. 2007; LópezÚbeda et al. 2015) the possible mechanisms of communication between spermatozoa and maternal reproductive tract
Table 2 Top six enriched KEGG pathways detected among 52 significantly upregulated genes (FDR $<0.05)$ in BOECs in response to non-contact co-culture with spermatozoa following $10 \mathrm{~h}$ of incubation.
Columns 'Gene IDs' and 'Gene count' show the significantly upregulated genes and the number of significantly upregulated genes that belong to the corresponding pathway

\begin{tabular}{lllll}
\hline Pathway ID & Pathway description & FDR & Gene IDs & Gene count \\
\hline bta04913 & Ovarian steroidogenesis & 0.003 & CYP1A1, CYP1B1, PTGS2, PLA2G4A & 4 \\
bta00830 & Retinol metabolism & 0.012 & CYP1A1, DHRS3, UGT1A6 & 3 \\
bta05204 & Chemical carcinogenesis & 0.012 & CYP1A1, CYP1B1, PTGS2, UGT1A6 & 4 \\
bta00140 & Steroid hormone biosynthesis & 0.013 & CYP1A1, CYP1B1, UGT1A6 & 3 \\
bta00980 & Metabolism of xenobiotics by cytochrome P450 & 0.039 & CYP1A1, CYP1B1, UGT1A6 & 3 \\
bta04060 & Cytokine-cytokine receptor interaction & 0.039 & TNFRSF11B, GDF15, TNFSF15, CXCR4 & 4 \\
\hline
\end{tabular}


Fig. 5 Quantitative real-time PCR-derived relative expression values of four genes in BOECs under control conditions (black, solid lines) and in response to contact co-culture with spermatozoa (blue, dashed lines) or noncontact co-culture with spermatozoa (orange, dotted lines).

Samples were collected after 30 , 90,150 , and 240 min of incubation. Each point represents an individual primary cell culture, with point shapes representing the experimental batch from which the sample was derived (circles: batch 1, triangles: batch 2, diamonds: batch 3). Relative expression values were calculated using the $2^{-\Delta \Delta-\mathrm{CT}}$ method
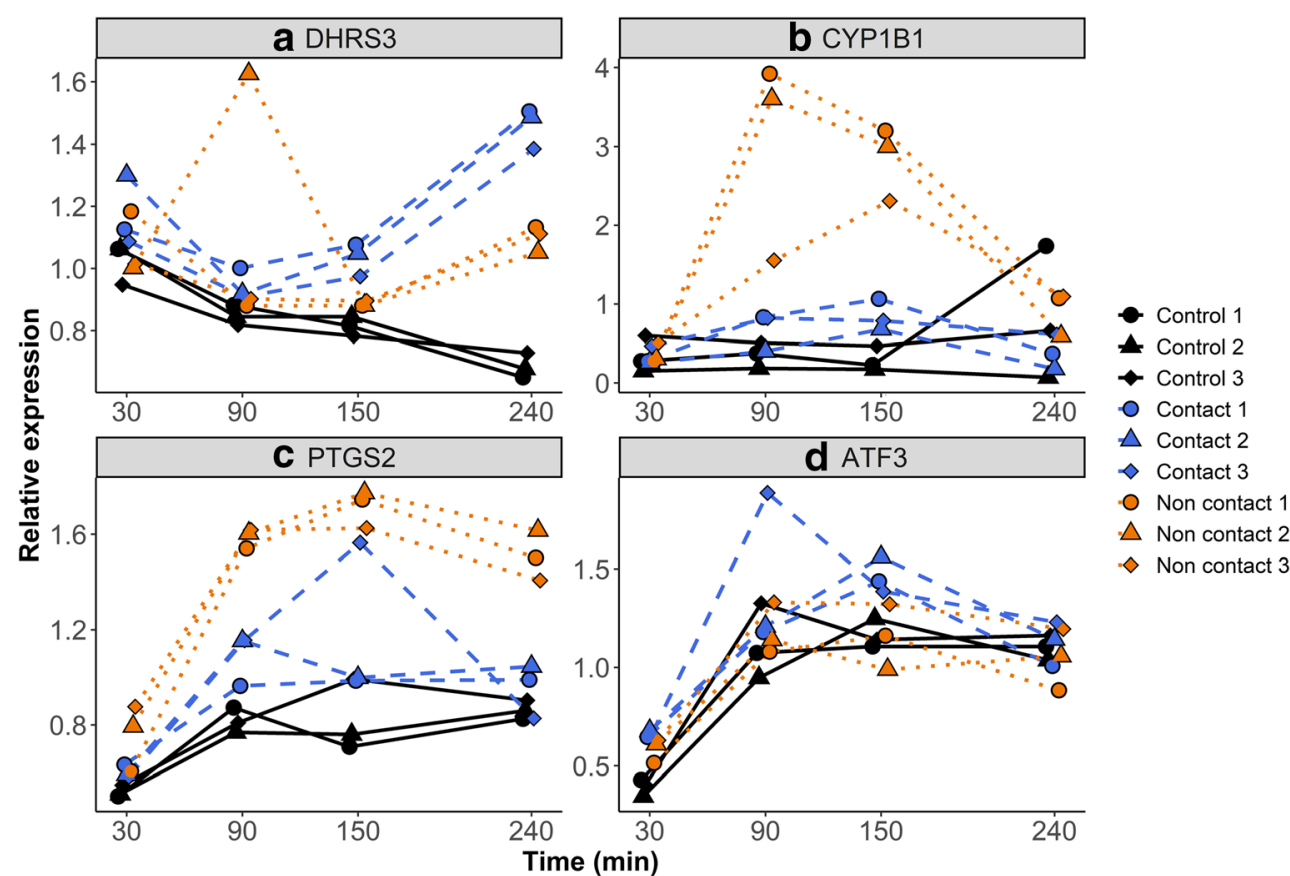

have been relatively under-explored. In this study, we tested whether spermatozoa are capable of communicating remotely with maternal cells by characterising the transcriptomic responses in BOECs incubated either in direct contact with spermatozoa or in a non-contact co-culture system as in general illustrated in Fig. 6.

Remarkably, not only were spermatozoa capable of inducing alterations to BOECs gene expression in the absence of direct physical contact, but the extent of gene expression changes was substantially greater than that induced by direct contact with spermatozoa (108 DEGs in response to noncontact co-culture compared to only $10 \mathrm{DEGs}$ in response to contact co-culture). This implies the existence of molecular signals released by the spermatozoa which are capable of evoking functional responses in the maternal tract. Nonetheless, we also found a small number of genes (e.g.

\section{Contact co-culture of sperms and BOEC's}

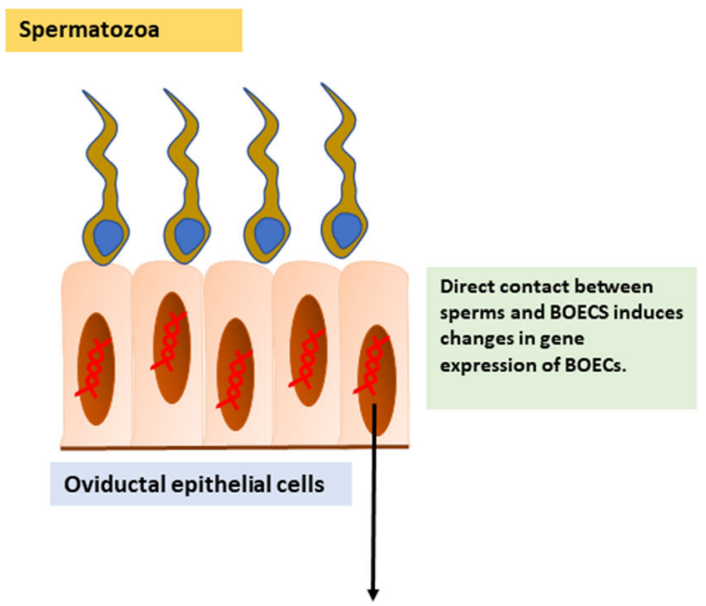

10 differentially expressed genes compared to control

\section{Non-contact co-culture of sperms and BOEC's}

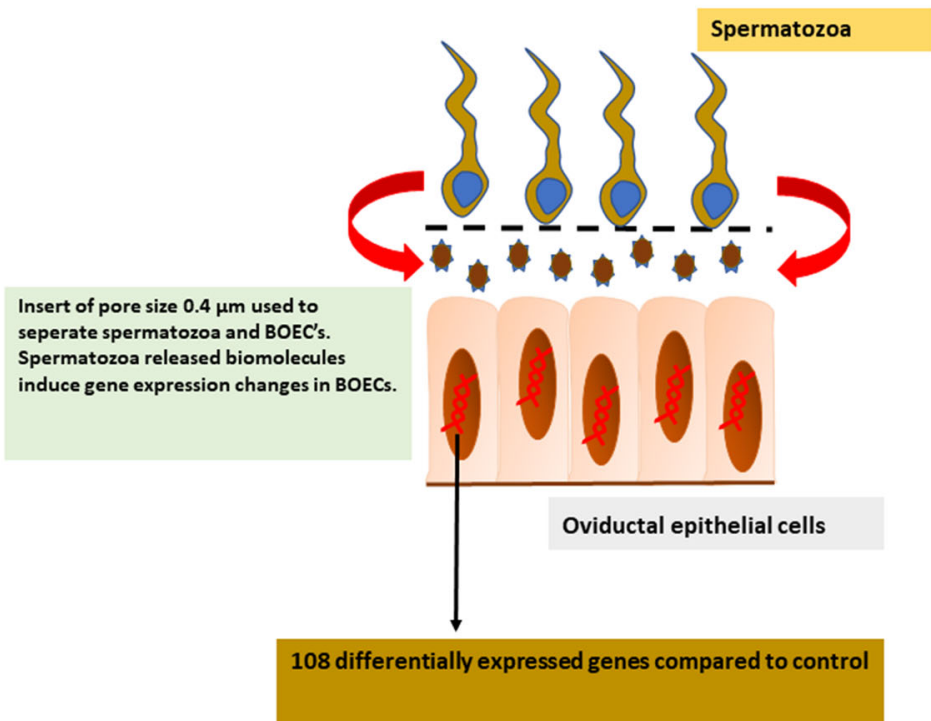

Fig. 6 Overall layout and findings of the study, where spermatozoa are capable of inducing gene expression changes in oviductal epithelial cells but the gene expression levels are highly dependent on the type of co-culture system 
ATF3) that were differentially expressed only in response to contact co-culture. Thus, it can be inferred that some responses require direct contact between spermatozoa and oviductal cells.

One hypothesis to explain the greater magnitude of differentially expressed genes in BOECs in non-contact compared to contact co-culture systems after $10 \mathrm{~h}$ of incubation is that the responses induced by direct contact may be more immediate than responses induced by non-contact. Conveyed by mediators of intercellular communication, the signals in noncontact system would take more time to reach the target cells, and therefore may subside after a longer time period even if the initial responses are of equal magnitude. Indeed, the expression levels of CYP1B1 and PTGS2, which were not affected by contact co-culture based on RNAseq data, were nonetheless increased in response to contact co-culture during the 4-h observation period of the qPCR experiment. However, the expression levels of both of these genes were considerably lower compared to the upregulation induced by non-contact culture which was highly pronounced even within this short timeframe. Assuming that the signals presented to BOECs through the non-contact system are also present in contact co-culture, both RNAseq and qPCR results suggest that signals released from the spermatozoa surface have strong influences on BOECs gene expression. However, the observed smaller number of differentially expressed genes in the contact co-culture system also suggests that many responses could be suppressed by ligands present only on the sperm surface. Indeed, $A T F 3$, which was upregulated in response to contact but not non-contact culture, has been known to have negative regulatory effects on the expression of PTGS2 during acute inflammation (Hellmann et al. 2015, p. 3). Inhibitory effects of ATF3 may therefore partly explain why PTGS2 upregulation was considerably weaker in response to contact culture compared to non-contact culture, as shown by the results of the time series experiment.

Results of the time series experiment revealed two genes involved in ovarian steroidogenesis pathway- PTGS2 and $C Y P 1 B 1$ - to be upregulated in both co-culture models within a short time frame. Prior studies have also shown that the gene expression of PTGS2 is upregulated in bovine oviductal epithelial cells in response to spermatozoa in a contact culture. It is also suggested that $P T G S 2$ enhances the oviductal ciliary motility and accelerates the transport of spermatozoa towards the oocyte (Kodithuwakku et al. 2007).

Regarding the putative functions of the responses to noncontact co-culture, pathway enrichment analysis revealed several pathways that were enriched with upregulated genes, the most enriched of which was the retinol metabolism pathway. Proper retinoic acid signalling is crucial for normal early embryo patterning and development. One of the key players in this pathway is DHRS3, which attenuates the synthesis of retinoic acid signalling (Kam et al. 2013, p. 3). Resulting from the time series experiment, we observed $D H R S 3$ to be significantly upregulated in both co-culture systems within a short timeframe (Fig. 4a). Ovarian steroidogenesis was also revealed as one of the significantly enriched pathways, which is pertinent as there is evidence that the steroid environment ensures successful oocyte fertilization (Yoshimura et al. 1986). The potential of the oviduct to secrete steroids has been demonstrated in a study conducted in porcine and has been suggested to have role in controlling early periods of pregnancy (Martyniak et al. 2018). Fertilization and early embryonic development are supported by secretion of oviductal factors, the regulation of which is controlled by the action of oviductal steriods (Ballester et al. 2014; Li and Winuthayanon 2017; Wollenhaupt et al. 1999). Ovarian steroids play a significant role in the synthesis of cytoplasmic factors that induce normal sperm head decondensation and formation of the male pronucleus (C 1977; Thibault et al. 1975). Defective sperm head decondensation has been associated as one of the factors responsible for the failed fertilization (Esterhuizen et al. 2002). The relevance of these pathways enriched in response to spermatozoa remains elusive, however the collective notion suggests that these pathways might have some role to play in terms of embryo development and fertilization. We assume that the count of spermatozoa being in millions is because not all the spermatozoa succeed in reaching the point of fertilisation, instead some of them die and some induce changes in the female reproductive tract and periconception milieu which are important with respect to successful fertilization.

Although our results indicate that some messenger biomolecules are released from spermatozoa, which in turn modulate the gene expression in BOECs independent of physical contact, more studies are required to biochemically characterize these messengers/bioactive agents released from spermatozoa that eventually lead to the observed alterations in gene expression. It would undoubtedly be insightful to pursue the identification of their specific composition and target receptors on BOECs along with the unfolding signalling mechanisms which ultimately lead to the change in the transcriptomic profile of the oviductal epithelial cells. Based on the current knowledge of mediators of intercellular communication, these signals could be mediated by proteins or cell-free nucleic acids, including those carried by extracellular vesicles. Notably, it has been suggested that many proteins and exosomes are bound to spermatozoa surface (Kasvandik et al. 2015; Samanta et al. 2018), some of which could mediate the effects observed in this study. Biochemical characterisation of signals released by spermatozoa may eventually contribute to better diagnostics and treatment for male infertility problems of various aetiology.

While our study gives a general idea of temporal gene expression alterations, we note that we did not bridge the gap between 4-h responses (qPCR) and 10-h responses (RNAseq), the extent to which short-term responses differ 
from long-term responses remains unclear. Therefore, in future a long-term time-course analysis should be taken into consideration. There is abundant room for further progress in determining how specific these signals are, from spermatozoa to oviductal epithelial cells and if these signals would be the same for any other cell-type.

Finally, we must acknowledge two potential confounding factors. Firstly, although the percoll gradient is likely to have removed the majority of dead spermatozoa and we observed that the majority of spermatozoa were motile following the percoll wash, we cannot rule out that some oviductal cell responses could have been elicited by membrane components released by damaged sperm. Secondly, the responses may also have been influenced by composition of the cryo-protectant extender (commonly used in semen straw preparations), which has been shown previously to influence specific parameters of the spermatozoon including calcium fluxes, viability and acrosome morphology. However, such an effect would have been similar in spermatozoa in direct and non-direct contact with oviductal cells (Graham and Foote 1987; Zhao and Buhr 1995).

\section{Conclusion}

Overall, the findings of our study shed new light on the communication between spermatozoa and the oviductal cells. Our results show for the first time that contact between spermatozoa and oviductal epithelial cells is not necessary to induce changes in the gene expression profile of the oviductal cells, suggesting that spermatozoa release signals that are capable of inducing alterations in gene expression profile of the recipient oviductal cells. Furthermore, we can deduce that the function of the spermatozoa is possibly not only confined to fertilization, but they may mediate other reproductive processes.

Acknowledgements Authors thank CellFit-European network of excellence (COST Action CA16119) for facilitating interaction and networking between the authors. Authors would also thank Annika Häling for her support during the laboratory work.

Author contributions Developed the concept, obtained funding and designed the experiment: Q.R, J.V, Ü.J, A.S, and A.F. Methodology: Q.R, J.V, K.G, M.H, K.J, M.N, A., A.F., Data analysis: Q.R, J.O, F.L. Writing manuscript: Q.R. J.V., J.O., F. L. Ü.J, A.S, and A.F., All authors read and approved the final manuscript.

Funding This work was supported by the European Union's Horizon 2020 research and innovation programme under grant agreement No 668989 (Transgeno), the Estonian Ministry of Education and research (grant IUT34-16); Enterprise Estonia (grant EU48695); the European Commission Horizon 2020 research and innovation programme under grant agreement 692,065 (project WIDENLIFE).

Data availability The sequencing data has been uploaded to the NCBI SRA repository under the BioProject ID: PRJNA624992.

\section{Compliance with ethical standards}

Competing interests Author(s) declared no conflict of interest.

Ethics approval The materials used for the experiments were offal obtained from the slaughter house from animals slaughtered to be used for human consumption. Semen straws were commercially available, for artificial insemination. No ethics approval was required to obtain these materials.

Consent to participate Not applicable.

Consent for publication Not applicable.

Code availability Not applicable.

Abbreviations BOECs, Bovine oviductal epithelial cells; EVs, Extracellular vesicles; DEGs, Differentially expressed genes; RNAseq, RNA sequencing

Open Access This article is licensed under a Creative Commons Attribution 4.0 International License, which permits use, sharing, adaptation, distribution and reproduction in any medium or format, as long as you give appropriate credit to the original author(s) and the source, provide a link to the Creative Commons licence, and indicate if changes were made. The images or other third party material in this article are included in the article's Creative Commons licence, unless indicated otherwise in a credit line to the material. If material is not included in the article's Creative Commons licence and your intended use is not permitted by statutory regulation or exceeds the permitted use, you will need to obtain permission directly from the copyright holder. To view a copy of this licence, visit http://creativecommons.org/licenses/by/4.0/.

\section{References}

Aalberts M, Sostaric E, Wubbolts R, Wauben MWM, Nolte-'t Hoen ENM, Gadella BM, Stout TAE, Stoorvogel W (2013) Spermatozoa recruit prostasomes in response to capacitation induction. BBA - Proteins Proteomics. https://doi.org/10.1016/j.bbapap. 2012.08.008

Aldarmahi A, Elliott S, Russell J, Fazeli A (2014) Effects of spermatozoaoviductal cell coincubation time and oviductal cell age on spermatozoa-oviduct interactions. Reprod Fertil Dev 26:358-365. https://doi.org/10.1071/RD12222

Almiñana C, Bauersachs S (2019) Extracellular vesicles in the oviduct: progress, challenges and implications for the reproductive success. Bioengineering 6:32. https://doi.org/10.3390/ bioengineering6020032

Alvarez-Rodriguez M, Atikuzzaman M, Venhoranta H, Wright D, Rodriguez-Martinez H (2019) Expression of immune regulatory genes in the porcine internal genital tract is differentially triggered by spermatozoa and seminal plasma. Int J Mol Sci 20

Ballester L, Romero-Aguirregomezcorta J, Soriano-Úbeda C, Matás C, Romar R, Coy P (2014) Timing of oviductal fluid collection, steroid concentrations, and sperm preservation method affect porcine in vitro fertilization efficiency. Fertil Steril 102:1762-1768.e1. https://doi.org/10.1016/j.fertnstert.2014.08.009

Bates D, Mächler M, Bolker B, Walker S (2015) Fitting linear mixedeffects models using lme4. J Stat Softw 67. https://doi.org/10.18637/ jss.v067.i01 
Benjamini Y, Hochberg Y (1995) Controlling the false discovery rate: a practical and powerful approach to multiple testing. J R Stat Soc Ser B Methodol 57:289-300

Bolger AM, Lohse M, Usadel B (2014) Trimmomatic: a flexible trimmer for Illumina sequence data. Bioinformatics 30:2114-2120. https:// doi.org/10.1093/bioinformatics/btu170

Brown J, Pirrung M, McCue LA (2017) FQC dashboard: integrates FastQC results into a web-based, interactive, and extensible FASTQ quality control tool. Bioinformatics 33:3137-3139. https://doi.org/10.1093/bioinformatics/btx373

C T (1977) Hammond Memorial Lecture. Are follicular maturation and oocyte maturation independent processes? J Reprod Fertil 51:1-15. https://doi.org/10.1530/jrf.0.0510001

Esterhuizen AD, Franken DR, Becker PJ, Lourens JGH, Müller II, Rooyen LHV (2002) Defective sperm decondensation: a cause for fertilization failure. Andrologia 34:1-7. https://doi.org/10.1046/j. 0303-4569.2001.00423.x

Fazeli A (2011) Maternal communication with gametes and embryo: a personal opinion. Reprod Domest Anim 46:75-78. https://doi.org/ 10.1111/j.1439-0531.2011.01870.x

Fazeli A, Affara NA, Hubank M, Holt WV (2004) Sperm-induced modification of the oviductal gene expression profile after natural insemination in mice. Biol Reprod 71:60-65. https://doi.org/10.1095/ biolreprod.103.026815

Ferraz M d AMM, Carothers A, Dahal R, Noonan MJ, Songsasen N (2019) Oviductal extracellular vesicles interact with the spermatozoon's head and mid-piece and improves its motility and fertilizing ability in the domestic cat. Sci Rep 9:9484. https://doi.org/ 10.1038/s41598-019-45857-x

Graham JK, Foote RH (1987) Effect of several lipids, fatty acyl chain length, and degree of unsaturation on the motility of bull spermatozoa after cold shock and freezing. Cryobiology 24:42-52. https:// doi.org/10.1016/0011-2240(87)90005-8

Hellmann J, Tang Y, Zhang MJ, Hai T, Bhatnagar A, Srivastava S, Spite M (2015) Atf3 negatively regulates Ptgs $2 / \operatorname{Cox} 2$ expression during acute inflammation. Prostaglandins Other Lipid Mediat 116-117: 49-56. https://doi.org/10.1016/j.prostaglandins.2015.01.001

Holt WV, Fazeli A (2016) Sperm selection in the female mammalian reproductive tract. Focus on the oviduct: hypotheses, mechanisms, and new opportunities. Theriogenology Swine Reproduction 85: 105-112. https://doi.org/10.1016/j.theriogenology.2015.07.019

Ireland JJ, Murphee RL, Coulson PB (1980) Accuracy of predicting stages of bovine estrous cycle by gross appearance of the corpus luteum. J Dairy Sci 63:155-160. https://doi.org/10.3168/jds.S00220302(80)82901-8

Jamaludin NA, Thurston LM, Witek KJ, Meikle A, Basatvat S, Elliott S, Hunt S, Andronowska A, Fazeli A (2019) Efficient isolation, biophysical characterisation and molecular composition of extracellular vesicles secreted by primary and immortalised cells of reproductive origin. Theriogenology 135:121-137. https://doi.org/10.1016/j. theriogenology.2019.06.002

Kam RKT, Shi W, Chan SO, Chen Y, Xu G, Lau CB-S, Fung KP, Chan WY, Zhao H (2013) Dhrs3 protein attenuates retinoic acid signaling and is required for early embryonic patterning. J Biol Chem 288: 31477-31487. https://doi.org/10.1074/jbc.M113.514984

Kasvandik S, Sillaste G, Velthut-Meikas A, Mikelsaar A-V, Hallap T, Padrik P, Tenson T, Jaakma Ü, Kõks S, Salumets A (2015) Bovine sperm plasma membrane proteomics through biotinylation and subcellular enrichment. Proteomics 15:1906-1920. https://doi.org/10. 1002/pmic.201400297

Kim D, Paggi JM, Park C, Bennett C, Salzberg SL (2019) Graph-based genome alignment and genotyping with HISAT2 and HISAT-genotype. Nat Biotechnol 37:907-915. https://doi.org/10.1038/s41587019-0201-4

Kodithuwakku SP, Miyamoto A, Wijayagunawardane MPB (2007) Spermatozoa stimulate prostaglandin synthesis and secretion in bovine oviductal epithelial cells. Reprod Camb Engl 133:10871094. https://doi.org/10.1530/REP-06-0201

Kornilov R, Puhka M, Mannerström B, Hiidenmaa H, Peltoniemi H, Siljander P, Seppänen-Kaijansinkko R, Kaur S (2018) Efficient ultrafiltration-based protocol to deplete extracellular vesicles from fetal bovine serum. J Extracell Vesicles 7. https://doi.org/10.1080/ 20013078.2017.1422674

Li S, Winuthayanon W (2017) Oviduct: roles in fertilization and early embryo development. J Endocrinol 232:R1-R26. https://doi.org/10. 1530/JOE-16-0302

Liao Y, Smyth GK, Shi W (2014) featureCounts: an efficient general purpose program for assigning sequence reads to genomic features. Bioinformatics 30:923-930. https://doi.org/10.1093/bioinformatics/ btt656

Long EL, Sonstegard TS, Long JA, Van Tassell CP, Zuelke KA (2003) Serial analysis of gene expression in Turkey sperm storage tubules in the presence and absence of resident sperm. Biol Reprod 69:469 474. https://doi.org/10.1095/biolreprod.102.015172

López-Úbeda R, García-Vázquez FA, Romar R, Gadea J, Muñoz M, Hunter RHF, Coy P (2015) Oviductal Transcriptome is modified after insemination during spontaneous ovulation in the sow. PLoS One 10:e130128. https://doi.org/10.1371/journal.pone.0130128

Martyniak M, Franczak A, Kotwica G (2018) Synthesis of steroid hormones in the porcine oviduct during early pregnancy. Reprod Biol 18:143-150. https://doi.org/10.1016/j.repbio.2018.02.002

Picelli S, Faridani OR, Björklund AK, Winberg G, Sagasser S, Sandberg R (2014) Full-length RNA-seq from single cells using smart-seq2. Nat Protoc 9:171-181. https://doi.org/10.1038/nprot.2014.006

Robinson MD, McCarthy DJ, Smyth GK (2010) edgeR: a bioconductor package for differential expression analysis of digital gene expression data. Bioinformatics 26:139-140. https://doi.org/10.1093/ bioinformatics/btp616

Rodríguez-Martínez H, Kvist U, Saravia F, Wallgren M, Johannisson A, Sanz L, Peña FJ, Martínez EA, Roca J, Vázquez JM, Calvete JJ (2009) The physiological roles of the boar ejaculate. Soc Reprod Fertil Suppl 66:1-21

Rozeboom KJ, Troedsson MH, Crabo BG (1998) Characterization of uterine leukocyte infiltration in gilts after artificial insemination. $\mathrm{J}$ Reprod Fertil 114:195-199. https://doi.org/10.1530/jrf.0.1140195

Samanta L, Parida R, Dias TR, Agarwal A (2018) The enigmatic seminal plasma: a proteomics insight from ejaculation to fertilization. Reprod Biol Endocrinol 16:41. https://doi.org/10.1186/s12958018-0358-6

Schindelin J, Arganda-Carreras I, Frise E, Kaynig V, Longair M, Pietzsch T, Preibisch S, Rueden C, Saalfeld S, Schmid B, Tinevez J-Y, White DJ, Hartenstein V, Eliceiri K, Tomancak P, Cardona A (2012) Fiji: an open-source platform for biological-image analysis. Nat Methods 9:676-682. https://doi.org/10.1038/nmeth.2019

The fallopian tubes: Their role in fertility and infertility, by R.H.F Hunter; Springer-Verlag, Berlin, 1988, 191 pp., 55 Figs., 2 in color. $\$ 120$. https://doi.org/10.1002/mrd.1120230412

Thibault C, Gerard M, Menezo Y (1975) In vitro acquired ability of rabbit and cow oocyte to ensure sperm nucleus decondensation during fertilization (MPGF), in: Annales de Biologie Animale. Biochimie, Biophysique

Walter W, Sánchez-Cabo F, Ricote M (2015) GOplot: an R package for visually combining expression data with functional analysis. Bioinforma Oxf Engl 31:2912-2914. https://doi.org/10.1093/ bioinformatics/btv300

Wickham H (2009) ggplot2: elegant graphics for data analysis, use R! Springer-Verlag, New York. https://doi.org/10.1007/978-0-387-98141-3

Winer J, Jung CK, Shackel I, Williams PM (1999) Development and validation of real-time quantitative reverse transcriptase-polymerase chain reaction for monitoring gene expression in cardiac myocytes in vitro. Anal Biochem 270:41-49. https://doi.org/10.1006/abio.1999.4085

Wollenhaupt K, Einspanier R, Gabler C, Schneider F, Kanitz W, Brüssow KP (1999) Identification of the EGF/EGF-R system in 
the oviduct and endometrium of pigs in early stages of pregnancy and early conceptus. Exp Clin Endocrinol Diabetes Off J Ger Soc Endocrinol Ger Diabetes Assoc 107:530-538. https://doi.org/10. 1055/s-0029-1232562

Yoshimura Y, Hosoi Y, Atlas SJ, Bongiovanni AM, Santulli R, Wallach EE (1986) The effect of ovarian steroidogenesis on ovulation and fertilizability in the in vitro perfused rabbit ovary. Biol Reprod 35: 943-948. https://doi.org/10.1095/biolreprod35.4.943

Yu G, Wang L-G, Han Y, He Q-Y (2012) clusterProfiler: an R package for comparing biological themes among gene clusters. OMICS J Integr Biol 16:284-287. https://doi.org/10.1089/omi.2011.0118
Zeisberg M, Neilson EG (2009) Biomarkers for epithelial-mesenchymal transitions. J Clin Invest 119:1429-1437. https://doi.org/10.1172/ JCI36183

Zhao Y, Buhr MM (1995) Cryopreservation extenders affect calcium flux in bovine spermatozoa during a temperature challenge. J Androl 16: 278-285. https://doi.org/10.1002/j.1939-4640.1995.tb00526.x

Publisher's note Springer Nature remains neutral with regard to jurisdictional claims in published maps and institutional affiliations. 